Annales Geophysicae (2001) 19: 1089-1094 (c) European Geophysical Society 2001

\title{
Magnetic field configuration and field-aligned acceleration of energetic ions during substorm onsets
}

\author{
A. Korth $^{1}$ and Z. Y. Pu ${ }^{2}$ \\ ${ }^{1}$ Max-Planck-Institut für Aeronomie, D-37191 Katlenburg-Lindau, Germany \\ ${ }^{2}$ Space Weather Laboratory, Center for Space Science and Application Research, Chinese Academy of Science, Beijing, \\ China and Department of Geophysics, Peking University, Beijing, China
}

Received: 1 March 2000 - Revised: 26 June 2001 - Accepted: 11 July 2001

\begin{abstract}
In this paper, we present an interpretation of the observed field-aligned acceleration events measured by GEOS-2 near the night-side synchronous orbit at substorm onsets (Chen et al., 2000). We show that field-aligned acceleration of ions (with pitch angle asymmetry) is closely related to strong short-lived electric fields in the $E_{y}$ direction. The acceleration is associated with either rapid dipolarization or further stretching of local magnetic field lines. Theoretical analysis suggests that a centrifugal mechanism is a likely candidate for the parallel energization. Equatorward or anti-equatorward energization occurs when the tail current sheet is thinner tailward or earthward of the spacecraft, respectively. The magnetic field topology leading to antiequatorward energization corresponds to a situation where the near-Earth tail undergoes further compression and the inner edge of the plasma sheet extends inwards as close as the night-side geosynchronous altitudes.
\end{abstract}

Key words. Magnetospheric physics (magnetospheric configuration and dynamics; plasma sheet; storms and substorms)

\section{Introduction}

Field-aligned energization of ions during the substorm expansion phase commonly occurs in the Earth's magnetotail and polar ionosphere (Quinn and McIlwain, 1979; Cladis, 1986; Mauk, 1986; Mauk and Meng, 1989, 1991; Delcourt et al., 1990, 1991; Perraut et al., 1993; Liu et al., 1994). For the past two decades, many studies have been devoted to reveal the acceleration processes. Quinn and Southwood (1982) first showed that the convection surge mechanism may result in short-lived accelerations of equatorial populations while they are rapidly injected earthward. Based on tracing guiding center trajectories, Mauk (1986) and Mauk and Meng (1989) found the effect on parallel energization during tail current disruption and possible creation of parallel electric

Correspondence to: A. Korth (korth@linmpi.mpg.de) potential drops. A good agreement between computed ion distributions and those measured at geosynchronous orbit was put forward. Furthermore, by performing single-particle simulations of ions during rapid dipolarization, Delcourt et al. (1990, 1991) and Delcourt (1993) demonstrated that the centrifugal mechanism has to be considered when the inductive electric field yields intense $\boldsymbol{E} \times \boldsymbol{B}$ drift and earthward injection of plasma sheet populations. It is seen in their simulations that ions are accelerated and decelerated when they are moving toward and away from the center plane of the plasma sheet, respectively, indicating that an equatorward parallel energization exits for ions along with their $\boldsymbol{E} \times \boldsymbol{B}$ drift. All of these investigations have given deep insight into the dynamical behaviour of ions in the near and mid-tail during substorm periods.

Furthermore, in $\mathrm{Pu}$ et al. (1998) and a recent paper by Chen et al. (2000), the pitch angle re-distribution (from symmetric to asymmetric) of energetic ions ( $>27 \mathrm{keV})$ was investigated at substorm onset by GEOS-2. The new findings in Chen et al. (2000) are as follows: (a) At substorm onset energetic ions were field-aligned accelerated either toward the equator (events 1 and 2) or away from the equator (events 3 and 4); (b) The field-aligned acceleration occurs while the local magnetic field lines rapidly dipolarize or stretch further tailward; (c) No apparent pitch angle asymmetry between $J(\alpha)$ and $J(\pi-\alpha)$ was found for energetic electrons, where $\alpha$ and $J(\alpha)$ represent the pitch angle of particles and the integral flux measured in that angle, respectively. This strongly suggests that the electric field parallel to the magnetic field was not the cause of the ion pitch angle redistribution.

In this paper, the aforementioned new findings are interpreted by examining the parallel motion of energetic ions near the night-side synchronous orbit. The original works by Delcourt et al. (1990) and by Mauk (1986) are extended by considering two types of magnetic field configurations characterized by an earthward or tailward declining equatorial curvature of field lines during the substorm onset phase. We will discuss these two characteristic types of field line configuration near the night-side geosynchronous orbit at substorm 
onsets and the relevant implications.

\section{Analysis of the parallel equation of motion}

\subsection{The equation of parallel motion}

In the presence of a time-varying magnetic field without a parallel electric field, the motion of charge particles parallel to the magnetic field can be described, within the first order approximation, by the following full parallel equation (Delcourt et al., 1990)

$$
\frac{d v_{\|}}{d t} \sim \boldsymbol{u}_{E} \cdot\left[\frac{\partial \boldsymbol{b}}{\partial t}+v_{\|} \frac{\partial \boldsymbol{b}}{\partial s}+\left(\boldsymbol{u}_{E} \cdot \nabla\right) \boldsymbol{b}\right]
$$

where $v_{\|}$is the velocity parallel to the magnetic field $\boldsymbol{B}$, and $s$ and $\boldsymbol{b}$ denote the distance and unit vector along the field line, respectively. For the sake of convenience, we adopted a coordinate system $(\boldsymbol{y}, \boldsymbol{b}, \boldsymbol{n})$, where $\boldsymbol{y}$ is the unit vector in the azimuthal direction, positive westward, $\boldsymbol{b}$ is the unit vector of the magnetic field $\boldsymbol{B}$, and $\boldsymbol{n}=\boldsymbol{y} \times \boldsymbol{b}$ completes the $(\boldsymbol{y}, b, n)$ triad. Using this coordinate system, one obtains

$$
\begin{aligned}
& \boldsymbol{E}_{y}=E_{y} \boldsymbol{y}, \\
& \boldsymbol{u}_{E}=\left[\boldsymbol{v}_{E} \cdot \boldsymbol{n}\right] \boldsymbol{n}=u_{E} \boldsymbol{n}, \\
& \frac{\partial \boldsymbol{b}}{\partial s}=\frac{\boldsymbol{n}}{R_{c}} \\
& \left(\boldsymbol{u}_{E} \cdot \nabla\right) \boldsymbol{b}=u_{E} \frac{\partial \boldsymbol{b}}{\partial n}
\end{aligned}
$$

where $\boldsymbol{v}_{E}$ represents the $\boldsymbol{E} \times \boldsymbol{B}$ drift velocity and $R_{c}$ is the local curvature radius of the field line $\left(R_{c}>0\right)$. The electric field component $E_{y}$ may be either positive or negative. For the four cases shown in Chen et al. (2000), $E_{y}$ was positive for event 3 and negative for event 1 during the period of interest (private communication of A. Pedersen). No electric field data were available for events 2 and 4.

The substorm-associated field-aligned acceleration events investigated in this paper were observed in the vicinity of a geostationary orbit not far from the midnight sector, and slightly off the magnetic equator $\left(<5.5^{\circ}\right.$ magnetic latitude). Therefore, the case of $E_{y}>0$ can well be described with the classical convection surge picture (Quinn and Southwood, 1982). In this situation, $\boldsymbol{u}_{E}=u_{E} \boldsymbol{n}$ will be southward (northward) and earthward in the northern (southern) hemisphere (see Fig. 1). On the other hand, if $E_{y}<0, \boldsymbol{u}_{E}$ will be northward (southward) and tailward in the northern (southern) hemisphere.

\subsection{Analysis of each term of Eq. (1)}

To understand why energetic ions may either be accelerated toward the equator or away from the equator, we first have to make quantitative estimations of each term on the right-hand side (RHS) of Eq. (1).

(a) The first RHS term is $\boldsymbol{u}_{E} \cdot \partial \boldsymbol{b} / \partial t$. For simplicity, we call this term the time dependent term $a_{t}$. As Delcourt et

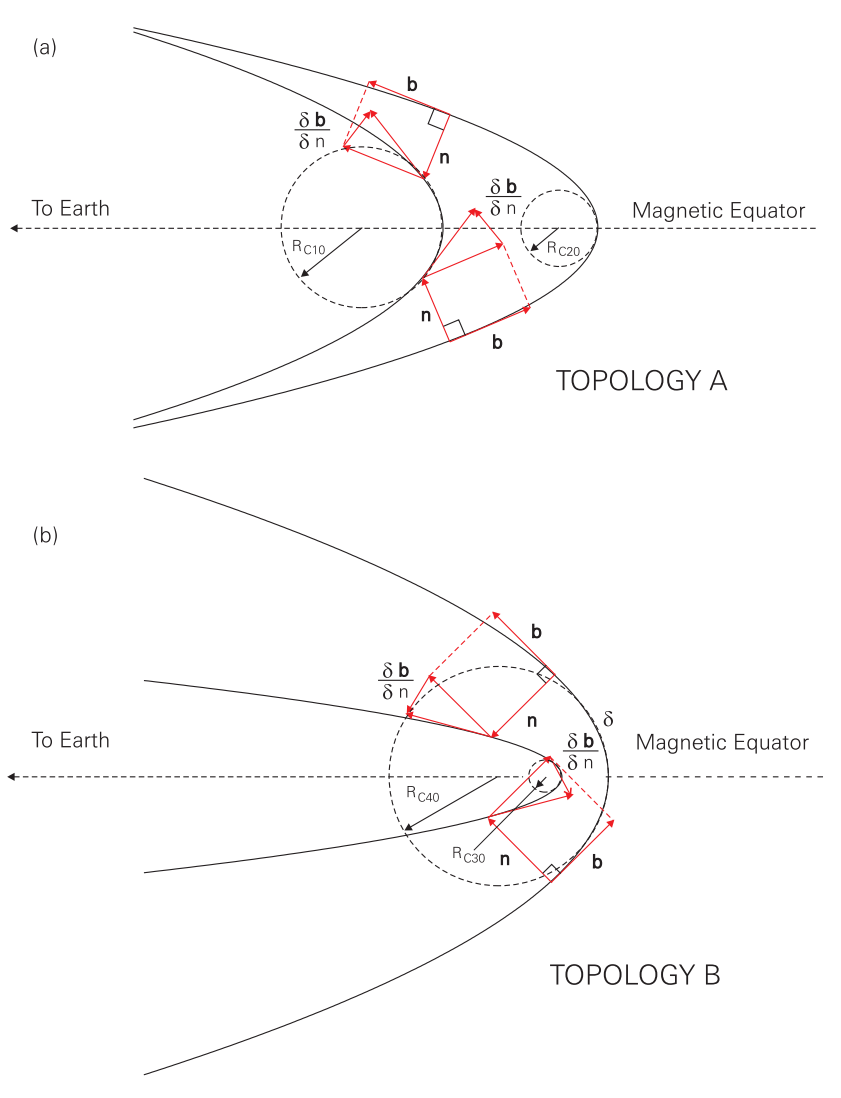

Fig. 1. Magnetic field configurations $\mathrm{A}$ and B. $R_{c 10}, R_{c 20}, R_{c 30}$, $R_{c 40}$ are the equatorial curvature radii of the magnetic field lines. $R_{c 10}>R_{c 20}$ and $R_{c 30}<R_{c 40}$. The direction of the vector $\partial \boldsymbol{b} / \partial n$ in (a) is different from that in (b). As a consequence, the sign of the centrifugal acceleration $a_{c \perp} \equiv \boldsymbol{u}_{E} \cdot\left[\left(\boldsymbol{u}_{E} \cdot \nabla\right) \boldsymbol{b}\right]=u_{E}^{2} \boldsymbol{n} \cdot \partial \boldsymbol{b} / \partial n$ in (a) is opposite to that in (b).

al. (1990) pointed out, this term is usually negligible with regard to the last two terms of Eq. (1). For instance, in the four field-aligned events, after the Pi2 onset, $\boldsymbol{b}$ changes less than $20^{\circ}$ during $1 \mathrm{~min}$. Suppose that during intense substorms near the night-side geosynchronous orbit, $u_{E} \sim 300 \mathrm{~km} / \mathrm{s}$ (Pu et al., 1998; Delcourt et al., 1990), one thus obtains $\boldsymbol{u}_{E} \cdot \partial \boldsymbol{b} / \partial t \sim 0.67 \pi v_{E} / 360 \sim 1.8 \cdot 10^{3} \mathrm{~m} / \mathrm{s}$. We will show later in this subsection that this is much smaller than the last RHS term of Eq. (1). On the other hand, in situ observations indicate that temporal variations of the magnetic field at substorm onsets are at times locally intense and $a_{t}$ may not always be neglected. It is easy to see that during field line dipolarization or tailward stretching, $a_{t}$ is $<0$ in the northern hemisphere and $>0$ in the southern hemisphere. This indicates that this term always leads to an equatorward acceleration for particles; hence it does not play a positive role in our events 3 and 4 in which ions were found to be accelerated away from the equator.

(b) The second RHS term or the centrifugal acceleration term is $a_{c \|}=\boldsymbol{u}_{E} \cdot v_{\|} \partial \boldsymbol{b} / \partial s$. Applying the coordinate system introduced above, we obtain $a_{c \|}=\boldsymbol{u}_{E} \cdot v_{\|} \partial \boldsymbol{b} / \partial \mathrm{s}$ $=u_{E} v_{\|} / R_{c}$. If $u_{E}>0, a_{c \|}$ has the same sign as $v_{\|}$, i.e. $(+)$ for the ions moving northward $\left(v_{\|}>0\right)$, and (-) for 
the ions moving southward $\left(v_{\|}<0\right)$. Therefore, $a_{c \|}$ accelerates all ions except those with a $90^{\circ}$ pitch angle, simply because $a_{c \|}=0$ if $v_{\|}=0$. Similarly, if $u_{E}<0, a_{c \|}$ decelerates all ions except those with a $90^{\circ}$ pitch angle. The acceleration/deceleration rate is the same for ions with the same magnitude of $v_{\|}$. In other words, although $a_{c \|}$ effectively energizes particles at the equator where the curvature of the field lines maximizes, it does not cause a pitch angle asymmetry with respect to $J(\alpha)$ and $J(\pi-\alpha)$ if the original distribution is symmetric, and hence, does not play a role in causing our pitch angle asymmetry events.

(c) The third RHS term or the additional centrifugal acceleration term is $a_{c \perp}=\boldsymbol{u}_{E} \cdot\left(\boldsymbol{u}_{E} \cdot \nabla\right) \boldsymbol{b}$. A simple derivation leads to

$a_{c \perp}=\boldsymbol{u}_{E} \cdot\left(\boldsymbol{u}_{E} \cdot \nabla\right)\left[\frac{\boldsymbol{B}}{B}\right]=$

$\boldsymbol{u}_{E} \cdot\left[\frac{u_{E}}{B} \frac{\partial \boldsymbol{B}}{\partial n}-\boldsymbol{B} \frac{u_{E}}{B^{2}} \frac{\partial B}{\partial n}\right] \sim \boldsymbol{u}_{E} \cdot\left[\frac{u_{E}}{B} \frac{\partial \boldsymbol{B}}{\partial n}\right] \sim \frac{u_{E}^{2}}{l}$,

where $l$ denotes the scale length of the magnetic field changes in the $\boldsymbol{n}$ direction. At expansion phase onset, the inductive $E_{y}$ yields intense (up to a few hundred $\mathrm{km} / \mathrm{s}$ ) $\boldsymbol{E} \times \boldsymbol{B}$ drift. Since during intense substorms near the night-side geosynchronous orbit, $l \sim 0.5 R_{E}$ (Pu et al., 1998; Delcourt et al., 1990), then we find $a_{c \perp}=u_{E}^{2} / l \sim 3 \cdot 10^{4} \mathrm{~m} / \mathrm{s}^{2}$, which is generally one order larger than $a_{t}$. One sees that under the consideration of $a_{c \perp}$, the increase of the parallel velocity can be as large as $1800 \mathrm{~km} / \mathrm{s}$ within $1 \mathrm{~min}$. Thus, the additional centrifugal acceleration $a_{c \perp}$ may contribute significantly to the particle parallel energization.

Furthermore, $a_{c \perp}=u_{E}^{2} \boldsymbol{n} \cdot \partial \boldsymbol{b} / \partial n$ does not depend upon the sign of $u_{E}$ and therefore not on the sign of $E_{y}$. The direction of the vector $\partial \boldsymbol{b} / \partial n$ is completely determined by the local magnetic field configuration. There are two possible types of field line configuration in the equatorial plane near the geosynchronous orbit. Topology A (Fig. 1a) is characterized by a tailward declining equatorial curvature radius of the magnetic field lines, while topology B (Fig. 1b) is characterized by an earthward declining equatorial curvature radius of the magnetic field lines. The direction of $\partial \boldsymbol{b} / \partial n$ in Fig. 1a is northward and tailward in the northern hemisphere, and equatorward and earthward in the southern hemisphere. Whereas in Fig. 1b, it is equatorward and earthward in the northern hemisphere, and southward and tailward in the southern hemisphere. Hence, the sign of $a_{c \perp}$ in Fig. 1a is $(-)$ in the northern hemisphere and $(+)$ in the southern hemisphere. While in Fig. 1b, the sign of $a_{c \perp}$ is $(+)$ in the northern hemisphere and (-) in the southern hemisphere. As a result, for topology A, $a_{c \perp}$ accelerates ions toward the equator, whereas in topology $\mathrm{B}, a_{c \perp}$ accelerates particles away from the equator.

Based on the aforementioned analysis, we then speculated that the magnetic fields near the night-side geosynchronous orbit in events 1, 2 and 3, 4 of Chen et al. (2000) probably appeared to be configuration A and B, respectively. In fact, the magnetic field geometry used by Delcourt et al. $(1990,1991)$

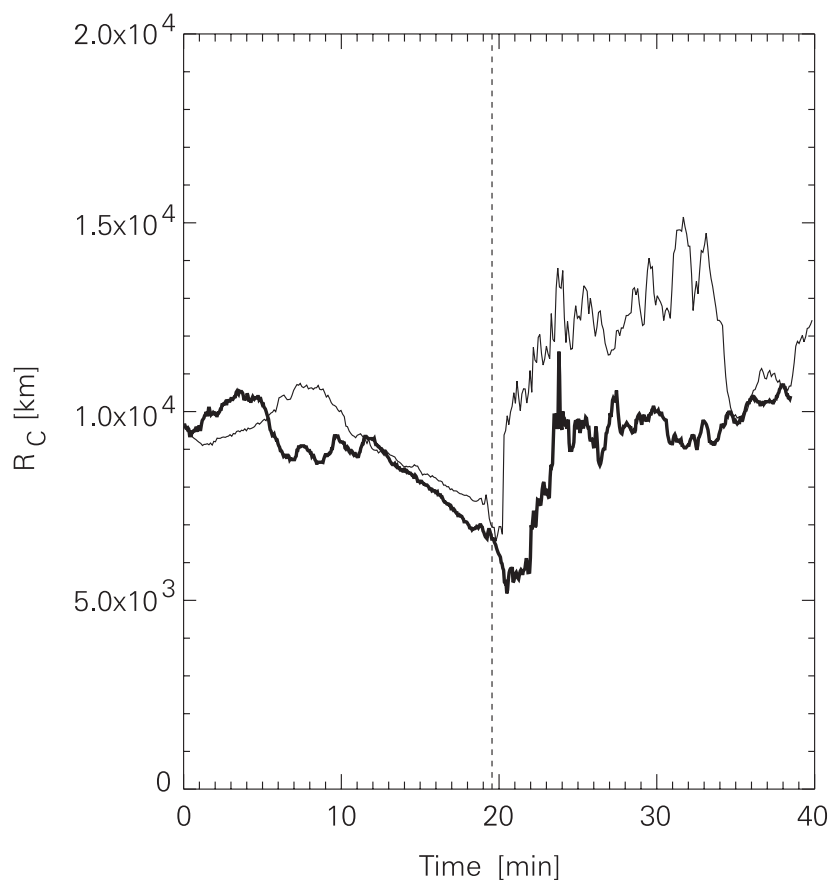

Fig. 2. The temporal variation of the equatorial curvature radii of the magnetic field lines threading AMPTE/CCE (thin line) and GOES-5 (thick line). The vertical dashed line marks the timing of the increase in the $H$ component at AMPTE/CCE. AMPTE/CCE was located tailward and very close to GOES-5.

in their simulations was topology A (see Fig. 3 of Delcourt et al., 1990).

In summary, the centrifugal energization $a_{c \perp}$ provides a likely mechanism responsible for equatorward and antiequatorward parallel accelerations of ions which were observed by Chen et al. (2000). We assume two types of magnetic field configuration (type A and B) which exist near the night-side geosynchronous orbit at substorm onsets.

\section{Discussions}

1. While the type A configuration of the magnetic field near the night-side synchronous orbit at substorm onsets is widely accepted, this does not preclude the existence of the configuration of type B. In fact, field line configuration B is often seen in physical and numerical magnetotail models (Kaufmann, 1987; Pulkkinen et al., 1991). It can also be derived from multi-satellite observations. For instance, on 30 July 1985, AMPTE/CCE was located tailward and very close to GOES-5. In situ measurements of three magnetic field components of the two spacecrafts were reported by Ohtani (1998). A calculation of the equatorial curvature radius of the magnetic field lines $\left(R_{c}\right)$ threading these two satellites has been made by using the magnetic field model by Quinn and McIlwain (1979) (with $q=4$ ). The results are plotted in Fig. 2 where the thin and thick lines indicate AMPTE/CCE and GOES-5, respectively. It is seen that prior to and at sub- 
storm onset, $R_{c}$ for the field line threading AMPTE/CCE was somewhat larger than that for the field line threading GOES5, though the AMPTE/CCE was situated tailward of GOES5. Later, after dipolarization in the expansion phase, the field line threading AMPTE/CCE is much more dipolar-like than that threading GOES-5, as commonly expected.

In topology B, the current sheet is thicker (thinner) in regions tailward (earthward) of the spacecraft. To create such a magnetic field configuration, the cross-tail currents in a region earthward of the satellite is required to be stronger than in the regions tailward of the satellite. This seems to be the case when the near-Earth tail undergoes further compression due to the braking of fast flows or enhanced magnetospheric convection, and the inner edge of the current sheet is tailward of, but not too far from, the geosynchronous orbit. In this situation, $B_{z}$ at the geosynchronous orbit may be weaker than that close to the inner edge of the current sheet (Shiokawa et al., 1997). It is known that this magnetic field configuration produces favorable conditions for the generation of the ballooning instability and triggering substorm dipolarization onsets (Roux et al., 1991; Pu et al., 1997, 1999, 2001).

2 . In the conventional convection surge picture, temporary intensification and subsequent relaxation of the westward $E_{y}$ coexists with an earthward displacement of the curved field lines when the field is being dipolarized. However, our observations (Chen et al., 2000) show that at or right after substorm Pi2 onset, the local magnetic field near the night-side synchronous orbit may shortly stretch out tailward, accompanied by a short-lived eastward $E_{y}$. At this moment, the centrifugal mechanism $a_{c \|}$ leads to parallel deceleration, and $a_{c \perp}$ causes the pitch angle asymmetry. This measurement provides further evidence that the growth phase signature and the expansion phase signature may coexist in the nearEarth magnetotail just after the onset time (Nagai, 1991). This is obviously not in contradiction to the observations that the tail current disruption is first initiated in a localized region (Arnoldy and Moore, 1983; Lui, 1978; Ohtani et al., 1991) and then expands tailward, earthward and azimuthally (Russell and McPherron, 1973; Rostoker et al., 1980; Nagai et al., 1987; Lopez and Lui, 1990; Ohtani et al., 1992; Ohtani 1998). This localization feature of substorm initiation has to be considered and requires more detailed studies for constructing a substorm expansion model.

3. It was thought that the geosynchronous region behaves approximately adiabatically during substorm dipolarization of which the characteristic time scale is about 1 to $2 \mathrm{~min}$ (Mauk and Meng, 1989). While the second adiabatic invariant of energetic ions is substantially violated, the magnetic moment $\mu$ would be basically conserved. The transport of charge particles in this region has been studied based on the guiding center trajectory tracing method (Mauk, 1986). On the other hand, magnetic field perturbations with a time scale comparable to the gyroperiod of ions may exist during the substorm expansion phase. A temporal nonadiabaticity occurs in this situation. Delcourt et al. (1990) thus developed the full parallel equation of motion to investigate the parallel motion of ions in the time-varying magnetic field during sub- storm times. In this paper, we apply this equation (Eq. (1) in the previous section) for our study and find that pitch angle asymmetry acceleration seen in GEOS-2 measurements can be explained in terms of the perpendicular centrifugal mechanism on the RHS of the equation.

Equation (1) is similar to the guiding center parallel equation (Northrop, 1963), except that it does not contain a term of $a_{g \|}=-\mu \partial B / \partial s$. This term represents the mirror force which diminishes at the magnetic equator, but rapidly increases at higher latitudes. It pushes particles toward the equator (trapping) and hence, obstructs the anti-equatorward acceleration. When $a_{g \|}$ dominates over the parallel motion of particles, the anti-equatorward energization will not appear as a noticeable phenomenon. Using the T-89 magnetic field model (Tsyganenko, 1989) with $K p=5$, for protons of $30 \mathrm{keV}$ and pitch angle $\alpha=30^{\circ}$ at $L=6.6$ and $\lambda=2.5^{\circ}$, we obtain $(\partial B / \partial s) / B \approx 5.2 \times 10^{-8} \mathrm{~m}^{-1}$ and $a_{g \|} \approx 2.6 \times 10^{4} \mathrm{~m} / \mathrm{s}^{2}$. The value for $a_{g \|}$ does not exceed the magnitude of the perpendicular centrifugal force $a_{c \perp} \sim 3 \times 10^{4} \mathrm{~m} / \mathrm{s}^{2}$ obtained in the previous section. Therefore, when using the guiding center approximation, one also obtains the anti-equatorward acceleration if the short-lived substorm electric field $E_{y}$ is large. In this paper, we focus our attention on the pitch angle asymmetry of ions related to the equatorward and anti-equatorward energization observed near the geosynchronous orbit. No noticeable pitch angle asymmetry of electrons was found by GEOS 2 close to substorm expansion onsets. This may be due to the small residence time that electrons spend while passing through the equatorial region with strong curved field line geometry. Nevertheless, in some substorms, electrons can experience parallel acceleration. As we can see in the bottom panel of event 4 in Chen et al. (2000), both $J\left(160^{\circ}<\alpha<180^{\circ}\right)$ and $J\left(10^{\circ}<\alpha<20^{\circ}\right)$ of electrons (not ions) clearly increased between 23:30 and 23:32 UT. Note that this flux enhancement leads to a pitch angle distribution anisotropy and not to a pitch angle asymmetry (with respect to $J(\alpha)$ and $J(\pi-\alpha)$ ), discussed in the present study and by Chen et al. (2000). We have not investigated this observation in detail. Probably the second RHS term of Eq. (1) can contribute to the generation of parallel energization of electrons which have a larger $v_{\|}$. It is worthwhile to recall that this centrifugal term accelerates particles with both $v_{\|}>0$ and $v_{\|}<0$ during dipolarization $\left(u_{E}>0\right)$ and hence, causes pitch angle anisotropy, but not pitch angle asymmetry.

\section{Summary and conclusions}

1. Field-aligned acceleration of ions which leads to an asymmetric pitch angle distribution at substorm onset is closely related to short-lived electric fields in the $E_{y}$ direction which are associated with either rapid dipolarization or further stretching of the local magnetic field lines.

2. This parallel energization is more noticeable when the substorm electric field $E_{y}$ becomes stronger. 
3. Centrifugal mechanism $\boldsymbol{u}_{E} \cdot\left(\boldsymbol{u}_{E} \cdot \nabla\right) \boldsymbol{b}$ appears to be the likely candidate responsible for the parallel energization of ions associated with the asymmetry of the pitch angle distribution.

4. Equatorward and anti-equatorward energization occurs, respectively, for the geomagnetic field configurations A and B. The topologies of the magnetic field near the night-side geosynchronous orbit in events 1,2 and 3,4 of Chen et al. (2000) are expected to be type B and A, respectively.

5. For configuration $\mathrm{B}$, the tail currents in a region earthward of the spacecraft are stronger than in a region tailward. This happens when the near-Earth tail undergoes a further compression and the inner edge of the plasma sheet extends close to the night-side geosynchronous altitude.

Acknowledgements. We thank Z. X. Chen for his original contribution to this work and R. W. H. Friedel and F. Frutos for fruitful discussions. The particle spectrometer on GEOS-2 was designed and constructed with support from the Max-Planck-Gesellschaft zur Förderung der Wissenschaften and the Bundesministerium für Forschung and Technologie through the DFVLR-PT under contract RV/4-B12/73(WRK243)-SF21. This work was also supported by NSFC (Grant No. 49984002 and 49834040) and the Chinese Key Research Project of G20000784. The authors are grateful to S. Ohtani for providing AMPTE/CCE and GOES-5 magnetic field data.

Topical Editor G. Chanteur thanks D. Delcourt and two other Referees for their help in evaluating this paper.

\section{References}

Arnoldy, R. L. and Moore, T. E., Longitudinal structure of substorm injections at synchronous orbit, J. Geophys. Res., 88, 6213, 1983.

Chen, Z. X., Korth, A., Pu Z. Y., and Mouikis, C. G., The pitch angle distribution of energetic ion fluxes at geosynchronous orbit around substorm onset, Geophys. Res. Lett., 27, 645, 2000.

Cladis, J. B., Parallel acceleration and transport of ions from polar ionosphere to plasma sheet, Geophys. Res. Lett., 13, 893-896, 1986.

Delcourt, D. C., Sauvaud, J. A., and Pedersen, A., Dynamics of single-particle orbits during substorm expansion phase, J. Geophys. Res., 95, 20 853, 1990.

Delcourt, D. C., Sauvaud, J. A., and Moore, T. E., Centrifugal flow reversal in the equatorial magnetosphere, in Auroral plasma dynamics, (Ed.) Lysak, R. L., Geophys. Monogr. 80, AGU, Washington D. C., 233, 1991.

Delcourt, D. C., The motion of near-Earth $(10 \sim 15 R e)$ plasma sheet particles during substorms, Adv. Space Res., 13, 237, 1993.

Kaufmann, R. L., Substorm currents: growth phase and onset, J. Geophys. Res., 92, 7471, 1987.

Liu, C., Perez, J. D., Moore, T. E., and Chappell, C. R., Low energy signature of substorm dipolarization, Geophys. Res. Lett., 21, 229-232, 1994.

Lopez, R. E. and Lui, A. T. Y., A multi-satellite case study of the expansion of a substorm current wedge in the near-Earth magnetotail, Geoophys. Res., 95, 8009, 1990.

Lui, A. T. Y., Estimates of current changes in the geomagnetotail associated with a substorm, Geophys. Res. Lett., 5, 853, 1978.
Mauk, B. H., Quantitative modeling of the "convection surge" mechanism of ion acceleration, J. Geophys. Res., 91, 13, 423, 1986.

Mauk, B. H. and Meng, C. I., Macroscopic magnetospheric particle acceleration, in Solar System Plasma Physics, (Eds.) Waite et al., Geophys. Monogr. 54, AGU, Washington D. C., 319, 1989.

Mauk, B. H. and Meng, C. I., The aurora and middle magnetospheric processes, in Auroral Physics, Cambridge University Press, (Eds.) Meng et al., 223, 1991.

Nagai, T., Singer, H. J., Ledley, B. G., and Olsen, R. C., Fieldaligned currents associated with substorms in the vicinity of synchronous orbit 1 . the 5 July 1979, substorm observed by SCATHA, GEOS 3, GEOS 2, J. Geophys. Res., 92, 2425, 1987.

Nagai. T., An empirical model of substorm-related magnetic field variations at synchronous orbit, in Magnetospheric Substorms, Geophys. Monogr. 64, (Eds.) Kan, J. R., Potemra, T. A., Kokubun, S., and Iijima, T., 91, AGU, Washington, D. C., 1991.

Northrop, T. G., The Adiabatic Motion of Charged Particles, Wiley Interscience, New York, 1963.

Ohtani, S., Takahashi, K., Zanetti, L. J., Potemra, T. A., McEntire, R. W., and Iijima, T., Tail current disruption in the geosynchronous region, in Magnetospheric Substorms, (Eds.) Kan, J. R., et al., Geophys. Monogr. 64, AGU, Washington, D. C., 131, 1991.

Ohtani, S., Kokubun, S., and Russell, C. T., Radial expansion of the tail current disruption during substorms: a new approach to the substorm onset region, J. Geophys. Res., 97, 3129, 1992.

Ohtani, S., Earthward expansion of tail current disruption: dualsatellite study, J. Geophys. Res., 103, 6815, 1998.

Perraut, S., Morane, A., Roux, A., Perdersen, A., Schmidt, R., Korth, A., Kremser, G., Aparicio, B., and Pellinen, R., Characterization of small-scale turbulence observed at substorm onsetsRelationship with parallel acceleration of particles, Adv. Space Res., 13, 217-222, 1993.

Pu, Z. Y., Korth, A., Chen, Z. X., Friedel, R. H. W., Zong, Q. G., Wang, X. M., Hong, M. H., Fu, S. Y., Liu, Z. X., and Pulkkinen, T. I., MHD drift Instability near the inner edge of the NECS and its application to substorm onset, J. Geophys. Res., 102, 14, 397, 1997.

Pu, Z. Y., Friedel, R. W. H., Korth, A., Zong, Q. G., Chen, Z. X., Roux, A., and Perraut, S., Evaluation of energetic particle parameters in the near-Earth magnetotail derived from flux asymmetry observation, Ann. Geophysicae, 16, 283, 1998.

Pu, Z. Y., Kang, K. B. , Korth, A., Fu, S. Y., Zong, Q. G., Chen, Z. X., Hong, M. N., Liu, Z. X., Mouikis, C. G., Friedel, R. W. H., and Pulkkinen, T., Ballooning instability in the presence of a plasma flow: A synthesis of tail reconnection and current disruption models for the initiation of substorms, J. Geophys. Res., 104, 10, 235, 1999.

Pu,Z. Y., Korth, A. , Chen, Z. X., Liu, Z. X., Fu, S. Y., Zong, G., Hong, M. H., and Wang, X. M., A Global Synthesis Model of Dipolarization at Substorm Expansion Onset, J. Atmospheric Solar-Terrestrial Physics, 63, 671-681, 2001.

Pulkkinen, T. I., Baker, D. N., Fairfield, D. H., Pellinen, R. J., Murphree, J. S., Elphinstone, R. D., McPherron, R. L., Fennell, J. F., Lopez, R. E., and Nagai, T., Modeling the growth phase of a substorm using the Tsyganenko model and multi-spacecraft observations: CDAW-9, Geophys. Res. Lett., 18, 1963, 1991.

Quinn, J. M. and McIlwain, C. E., Bouncing ion clusters in the Earth's magnetosphere, J. Geophys. Res., 74, 7365, 1979.

Quinn, J. M., and Southwood, D. J., Observations of parallel ion energization in the equatorial region, J. Geophys. Res., 87, 10, 
536, 1982.

Rostoker, G., Akasofu, S.-I., Forster, J., Greenwald, R. A., Kamide, Y., Kawasaki, K., Lui, A. T. Y., McPherron, R. L., and Russell, C. T., Magnetospheric substorms: Definition and signatures, J. Geophys. Res., 85, 1663, 1980.

Roux, A., Perraut, S., Robert, P., Morane, A., Pedersen, A., Korth, A., Kremser, G., Aparicio, B., Rodgers, D., and Pellinen, R., Plasma sheet instability related to the westward traveling surge,
J. Geophys. Res., 96, 17 697, 1991.

Russell, C. T. and McPherron, R. L., The magnetotail and substorms, Space Sci. Rev., 15, 205, 1973.

Shiokawa, K., Baumjohann, W., and Haerendel, G., Braking of high-speed in the near-Earth tail flows, Geophys. Res. Lett., 24, 1179-1192, 1997.

Tsyganenko, N. A., A magnetospheric magnetic field model with a warped tail current sheet, Planet. Space Sci., 37, 5, 1989. 